\title{
Light-Induced Bulk Architecturation of PDMS Membranes
}

\author{
Arthur Stricher, Renaud G. Rinaldi, Guilherme Machado, \\ Gregory Chagnon, Denis Favier, Laurent Chazeau, François Ganachaud*
}

One major challenge of biomaterial engineering is to mimic the mechanical properties of anisotropic, multifunctional natural soft tissues. Existing solutions toward controlled anisotropy include the use of oriented reinforcing fillers, with complicated interface issues, or UV-curing processing through patterned masks, that makes use of harmful photosensitive molecules. Here, a versatile process to manufacture biocompatible silicone elastomer membranes by light degradation of the platinum catalyst prior to thermal cross-linking is presented. The spatial control of network density is demonstrated by experimental and theoretical characterizations of the mechanical responses of patterned cross-linked membranes, with a view to mimic advanced implantable materials.

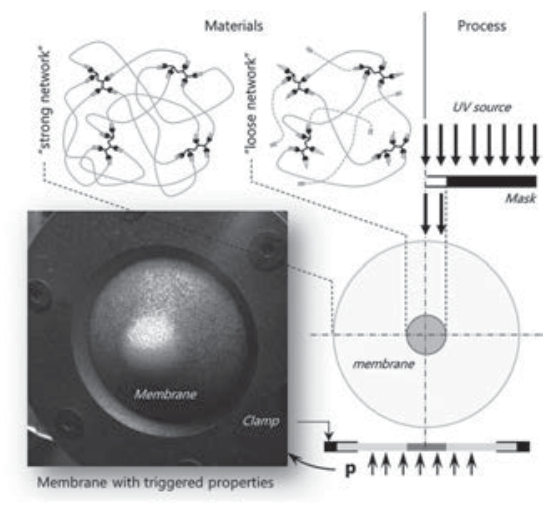

\section{Introduction}

Elastomeric materials are of great use for biomedical applications, ${ }^{[1]}$ providing properties close to the ones of natural soft tissues. More precisely, silicones are widespread, thanks to their biocompatibility, ease of sterilization, ${ }^{[2]}$ and their

\footnotetext{
Dr. A. Stricher, Dr. F. Ganachaud IMP, UMR CNRS 5223, INSA-Lyon Bâtiment Jules Verne 17 avenue Jean Capelle F69621 Villeurbanne Cedex, France E-mail: francois.ganachaud@insa-lyon.fr Dr. R. G. Rinaldi, Prof. L. Chazeau MATEIS, UMR CNRS 5510, INSA-Lyon Bâtiment Blaise Pascal 7 Avenue Jean Capelle F69621 Villeurbanne Cedex, France Dr. G. Machado Laboratoire de Mécanique et d'Acoustique CNRS - UPR 7051, 4 impasse Nikola Tesla CS 40006, F13453 Marseille Cedex, France Dr. G. Chagnon, Prof. D. Favier TIMC-IMAG, IN3S, Pavillon TAILLEFER

F38706 La Tronche Cedex, France
}

ability to sustain large deformation. Aside from mammal implants, silicones are used in many other applications, such as blood pumps, maxillofacial prosthesis, replacement esophagus, or artificial skin. ${ }^{[3]}$ A singular property of most natural soft tissues is their mechanical anisotropy, ${ }^{[4,5]}$ often related to the orientation of collagen fibers. ${ }^{[6]}$ To mimic this feature, anisotropic rubbers can be made by the introduction of oriented fibers, ${ }^{[7,8]}$ the controlled diffusion of reinforcing fillers ${ }^{[9]}$ or species. ${ }^{[10-12]}$ More straightforward routes include thickness variation ${ }^{[13,14]}$ and oriented damaging of the network. ${ }^{[15,16]}$ The limitations are however multiple: in the case of fibers, the incorporation of interfaces (and therefore potential defects) becomes an issue. As far as "diffusion" is concerned, fine control of the process is lacking, while thickness control requires specially crafted molds and highly uneven surfaces. Thus, locally controlling the cross-linking density of the network is a legit option, since it suppresses interfaces problems and allows for any mechanical pattern. Some studies investigated the addition of photo-cross-linking initiators ${ }^{[10]}$ or inhibitors, ${ }^{[17-19]}$ which allows a great versatility, considering UV rays responsible for cross-linking (or its inhibition) can be readily localized. However, they require the addition of harmful molecules and involve chemical reactions besides 
regular silicone cross-linking, which may involve unwanted byproducts and/or side reactions.

Here, we introduce a new method allowing for the spatial control of the cross-linking density of any additioncured silicone elastomers (e.g., liquid silicone rubbers, or LSR), without either additional chemical reaction or macromolecular network degradation. Curing of these elastomers results from the hydrosilylation reaction of long vinyl telechelic polydimethylsiloxane (PDMS) chains on hydride-bearing siloxane cross-linkers (Figure 1a). ${ }^{[20]}$ The reaction takes place in temperature in the presence of a catalyst, the most widespread being the Karstedt's catalyst. ${ }^{[21]}$ The process benefits from the photoinstability ${ }^{[22]}$ of this platinum complex: when irradiated in the presence of an UV-absorbing solvent, it gradually deteriorates into inert colloidal Pt(0). Therefore, the ultraviolet (UV) irradiation of a un-cross-linked LSR blend in presence of an aromatic solvent (here xylenes, vide infra) leads to the degradation of the Karstedt's catalyst, and hence to a reduction of the silicone cross-linking rate. The UV irradiation can be localized via the use of masks to block the rays, or laser devices to focus them. ${ }^{[23]}$ By doing so, the cross-linking kinetics can be adjusted spatially, and the reaction stopped before its completion by cooling and/or end-capping the remaining reactive groups, as depicted in Figure $1 b$. The resulting mechanical properties will only depend on the UV dose received, and consecutive hot cure parameters (temperature and duration).

\section{Results and Discussion}

Based on the aforementioned technique, the manufacturing of mechanically patterned elastomer membranes is obtained by the method depicted in Figure 1c. A mixture of two-part silicone (Silbione 4360AB from Bluestar Silicone) and xylenes is casted in a mold, irradiated, degassed, and finally hot cured. The curing time and

(a)

)

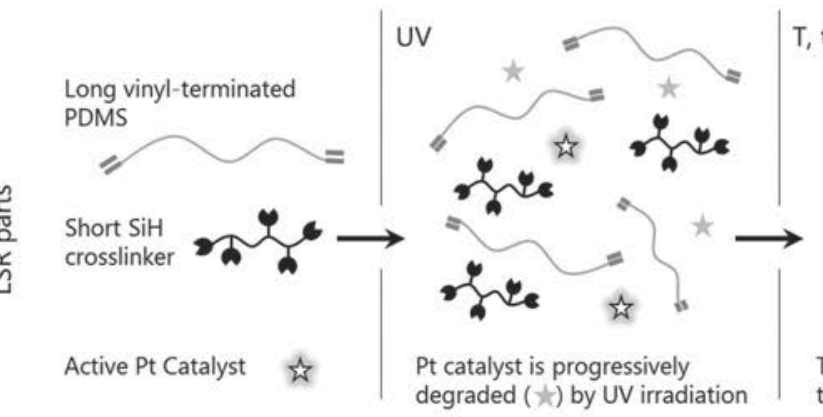

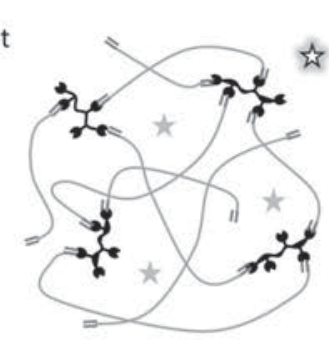

The network is less crosslinked, therefore softer

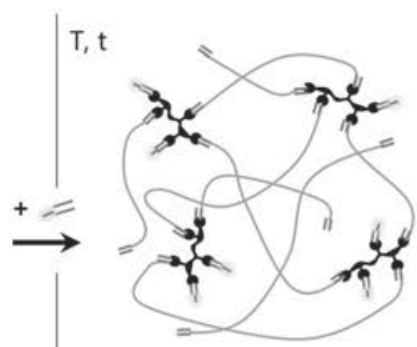

End-capping of remaining crosslinking sites

(b)
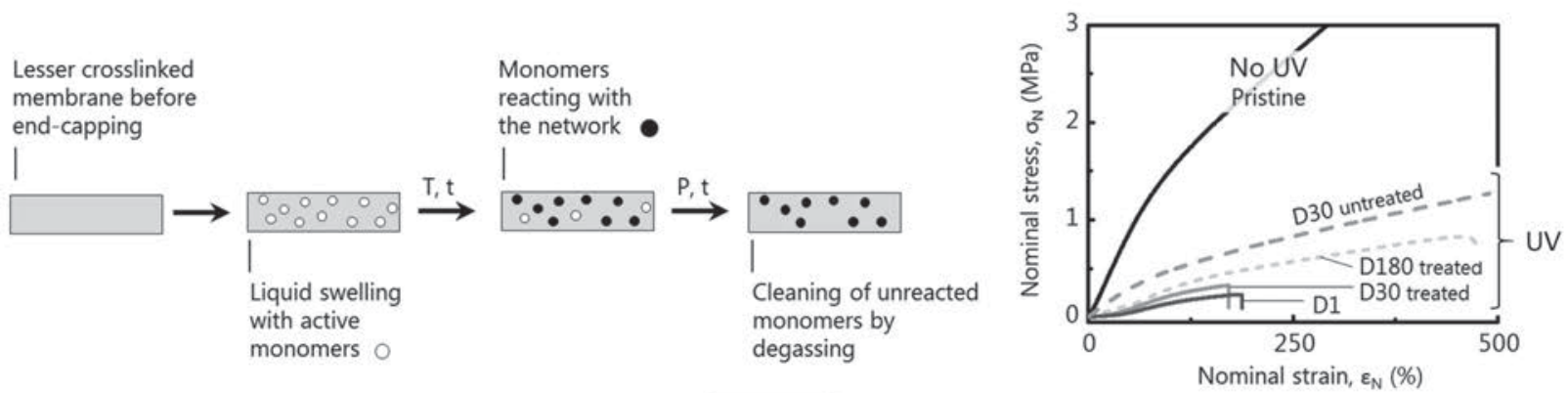

(c)

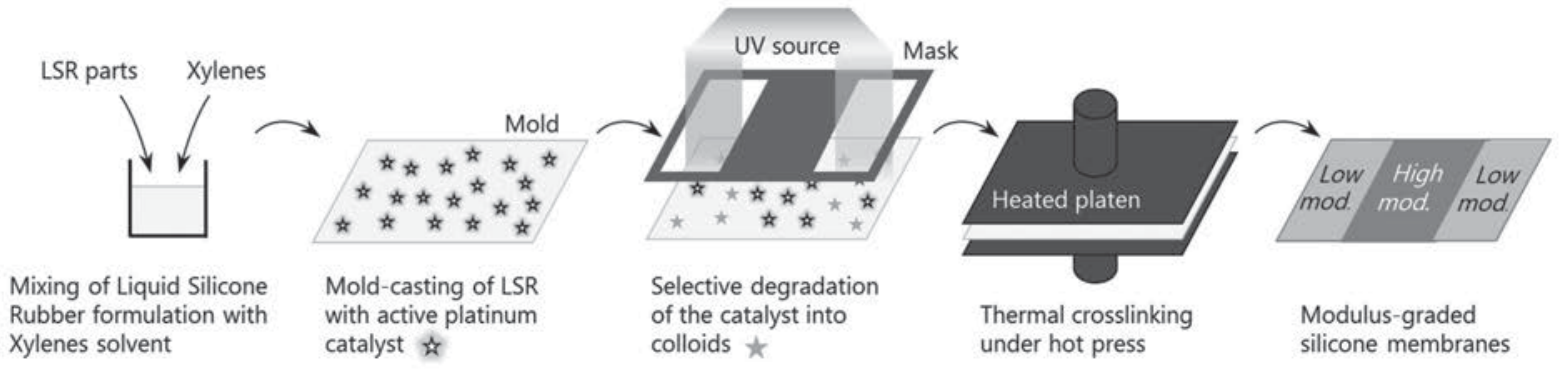

Figure 1. Control of cross-linking strategy: a) UV-degradation of the cross-linking density. b) Post-functionalization of irradiated zones, comprising hydride moieties available. Viability is demonstrated by the mechanical stabilization of under cross-linked membranes, and by 'H HR-MAS NMR study of the treated samples (Section S6, Supporting Information). c) Manufacturing of silicone membranes with local control of the mechanical properties. 
(a)

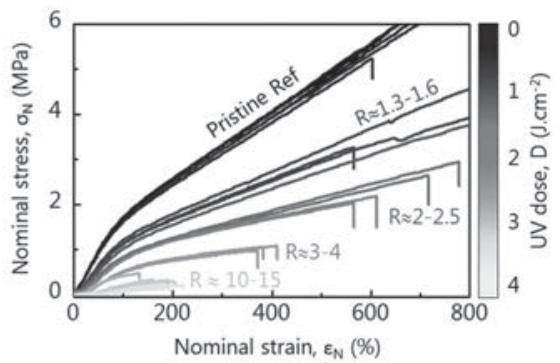

(c)

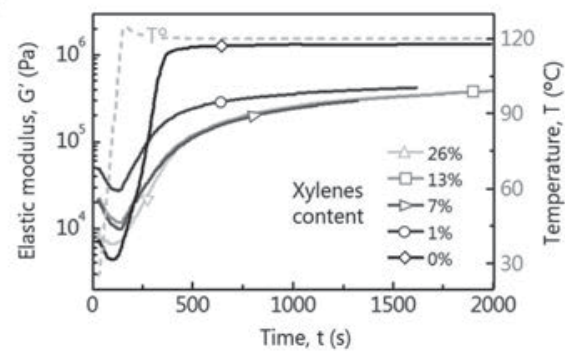

(b)

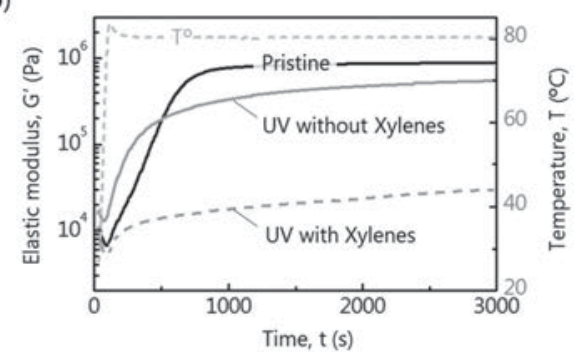

(d)
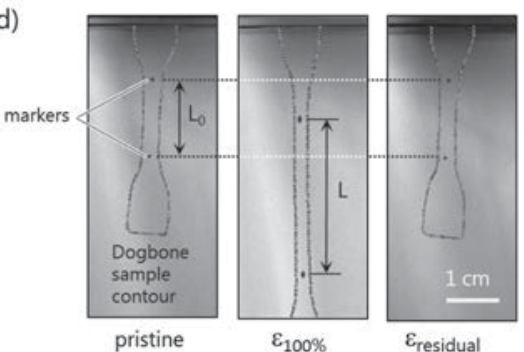

Figure 2. Characterization of fully irradiated materials: a) Mechanical tensile behavior versus UV dose. b) Cross-linking kinetics of a pristine and two irradiated samples with or without xylenes. c) Effect of the quantity of solvent during irradiation on the cross-linking kinetics. d) Negligible residual strain after a $100 \%$ stretch $\left(D=4 \mathrm{~J} \mathrm{~cm}^{-2}\right)$.

temperature are chosen so that the non-irradiated samples are fully cross-linked. The irradiation dose is tuned to trigger the mechanical behavior of the irradiated samples/ zones, as illustrated in Figure $2 \mathrm{a}$ where the nominal stressstrain uniaxial tension responses with varying irradiation time are displayed. The Young's modulus, stress, and strain at break decrease with increasing irradiation dose (from 0 to $\left.4.5 \mathrm{~J} \mathrm{~cm}^{-2}\right) . R=\sigma_{100}^{\mathrm{ref}} / \sigma_{100}^{\mathrm{UV}}$ is then calculated to quantify the difference in stress magnitude at a $100 \%$ strain value between the irradiated and the non-irradiated (reference) samples. $R$ is here as high as 15, which is the highest value reported in the literature for silicone elastomers without varying prepolymer length nor composition, ${ }^{[10,11,19]}$ membrane thickness, ${ }^{[12,24]}$ nor adding any photo-activated molecules. ${ }^{[10,18,25]}$ Since the determination of the elastic modulus from tensile test is not trivial in elastomeric materials, ${ }^{[26]}$ stress-strain plots were fitted with the neo-Hookean model in order to estimate the decrease of mechanical properties with respect to the UV dose. Interestingly, the decrease of the fitting constant is almost linear with an increasing dose, as shown in Section S1 (Supporting Information).

The observed drop in the deformation at break, yet remaining larger than $200 \%$ whatever the irradiation dose assigned, results from the fact that the macromolecular network of the irradiated silicone is mixed with a large amount of free chains (the amount of extractable material as a function of the UV-dose can be found in Section S2, Supporting Information) which decreases its resistance to tear and to high strains.

To ensure that the observed effect was not due to polymer photodegradation, polysiloxanes contained in the used LSR formulation were studied by nuclear magnetic resonance (NMR) spectroscopy and size exclusion chromatography (SEC), before and after irradiation (Section S3, Supporting Information). The results showed that the irradiation did not lead to any relevant change in molar mass or in the content of reactive moieties whether or not an aromatic solvent was present during the irradiation.

Silicone materials were then manufactured after irradiating only one of the two parts of the LSR formulation in the presence of xylenes. Both parts contain silica and vinyl functionalized oil while only one encloses the catalyst. ${ }^{[27]}$ As shown in Section S4 (Supporting Information), when the part which does not contain any catalyst is irradiated, the resulting material reaches the macroscopic properties of the fully un-irradiated one. Conversely, the material obtained from the irradiation of the part comprising the catalyst exhibits mechanical properties equivalent to the fully irradiated sample, confirming the key role of the catalyst degradation.

Cross-linking kinetics of irradiated LSR was therefore studied during an isotherm using a plane/plane rheometer (Figure 2b,c). Upon curing, the silicone oil transforms into a cross-linked elastomer, which is accompanied by an increase of its storage modulus by several orders of magnitude. The cross-linking of the pristine LSR takes place in a few minutes at $90{ }^{\circ} \mathrm{C}$, while it requires several hours when previously irradiated in the presence of xylenes. Interestingly enough, when irradiation was performed in presence of a non-aromatic solvent, such as methylcyclohexane, no effect on the reaction kinetics was noticed (Section S5, Supporting Information). This shows 
the important role of a UV-absorbing solvent that transfers UV energy to the catalyst, leading to the degradation of its labile ligands and platinum aggregation. Similar effect has been reported on other platinum or metallic complexes. ${ }^{[28,29]}$ The effect of the quantity of xylenes during the irradiation has been investigated as shown in Figure 2c: the irradiation of the silicone formulation alone leads to the same cross-linking behavior as the nonirradiated one, and the presence of a quantity as low as $1 \%$ (in mass) of xylenes during irradiation is sufficient to deeply affect the cross-linking kinetics.

To further study the photo-degradation mechanism of the catalyst, which has not been clearly reported in the literature, Karstedt's catalyst (in an excess of divinyl tetramethyl disiloxane, which is inert to UV) was irradiated at a wavelength $\lambda=254 \mathrm{~nm}$ in the presence of an aromatic solvent. Throughout irradiation, the solution color turned from transparent to brown attributed to colloidal platinum formation. ${ }^{[28]}$ Colloids were indeed observed by imaging this mixture in transmission electron microscope (TEM), as seen in Section S6 (Supporting Information). Bibliography indicates that such colloids are also formed during the regular hydrosilylation reaction, becoming an inert, transformed state of the catalyst. ${ }^{[30,31]}$ When photoirradiated prior to curing, Karstedt's catalyst most likely degrades into platinum colloids and therefore cannot properly catalyze the cross-linking reaction of LSRs. The reduction of the cross-linking kinetics of irradiated sample was also demonstrated with the Speier's catalyst and a carbene-platinum-based catalyst (Section S7, Supporting Information). These important results suggest that the presented process can be adapted to a wide range of silicone formulations to produce biocompatible membranes without the use of photo-initiators.

Affecting the cross-linking kinetics rather than blocking it completely means that ultimately, provided that few active catalyst remains, almost full cross-linking of irradiated zones can still be achieved by bringing enough time and heat to the material. Chemical reactions can therefore be performed on the remaining crosslinking sites to functionalize, or to stabilize the material after curing, as depicted in Figure 1a,c. Mechanical stabilization of a membrane for $30 \mathrm{~d}$ was achieved by endcapping cross-linking sites with monofunctional vinyl silanes with remaining hydride groups of the cross-linker. High-resolution magic angle spinning nuclear magnetic resonance (HR-MAS NMR) spectroscopy, suitable for gels and lightly cured elastomers, allowed to monitor the reaction and evidenced the consuming of $\mathrm{Si}-\mathrm{H}$ while $\mathrm{Si}-\mathrm{Vi}$ moieties remained unchanged (Section S8, Supporting Information). When samples were aged for a longer time (six months), they displayed a slight increase of mechanical properties. Full mechanical stabilization might still be achieved either by forcing the reaction with vinyltrimethylsilane or VTMS (longer post curing times) or by quenching the remaining active platinum with sulfur compounds. ${ }^{[32]}$

The elastic recovery of stretched materials produced by our method was then studied in order to evaluate the influence of the reduced cross-linking on their elasticity, which is crucial when targeting biological applications. To do so, samples were stretched up to $100 \%$ strain, then released, and the elastic return was measured $10 \mathrm{~s}$ after the release. Regardless of the cross-linking state, samples displayed less than $3 \%$ of residual strain at the first stretch, and less than $1 \%$ for the following cycles, pictured in Figure 2d. Irradiated samples seemed to take longer time to relax and an attempt to quantify the difference of relaxation is described in Section s9 (Supporting Information).

Dynamical mechanical analysis provides the evolution of the storage modulus and damping factor versus frequency (for a given temperature) of the manufactured elastomers as displayed in Section S10 (Supporting Information). For the case of perfectly (entropic) hyperplastic materials, these properties are supposed to be insensitive to the loading rates. Nevertheless, the modulus and damping factor of the present silicones increase with increasing frequency, evidencing some viscoelastic contribution. More importantly, the damping is significantly greater in the irradiated sample (the damping factor is multiplied by 3 with $R=6$ ), a consistent result since its network comprises a larger amount of unreacted and dangling chains. This difference of viscoelastic behavior can be used as an advantage, for instance, for applications where damping has to be localized in prescribed regions.

The patterning/architecturation of a membrane can be finally obtained through the use of a mask positioned between the UV source and the LSR mix, resulting in a spatial control of the irradiation. Practically, the non afocal UV source and a gap between the polymer and the mask suggest that a transition zone, or an interphase, may exist between the hard and soft phases of patterned elastomers where the modulus is likely to vary gradually. The characterization of such interphase is not easy, yet important. Indeed, a sharp interphase, i.e., a brutal change in the material mechanical properties, would result in stress concentration leading to early breakage of the sample. Moreover, the variation in material properties may provoke complex stress and strain fields in the transition zone even for simple (uniaxial) loading conditions. In order to control the strain field and thus proceed to the interphase macroscopic characterization, a bulge test was set. ${ }^{[3]}$ The tested sample displays a circular under-cross-linked ( $r=5 \mathrm{~mm}$ ) disk within a normally cross-linked matrix, as depicted in Figure 3a, and Movie S1 (Supporting Information). The membrane is clamped so that the center of the irradiated disk is centered 
(a)

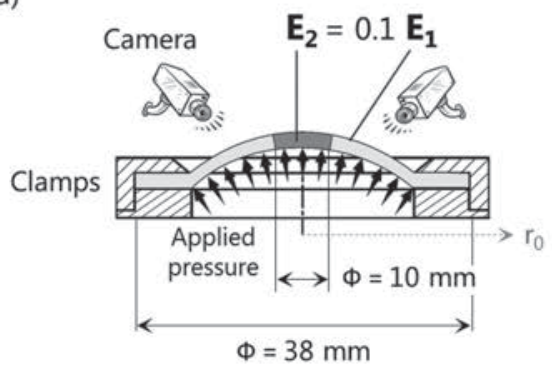

(b)

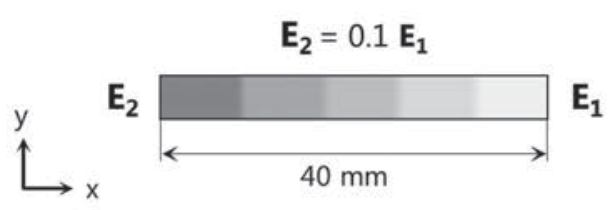

(c)

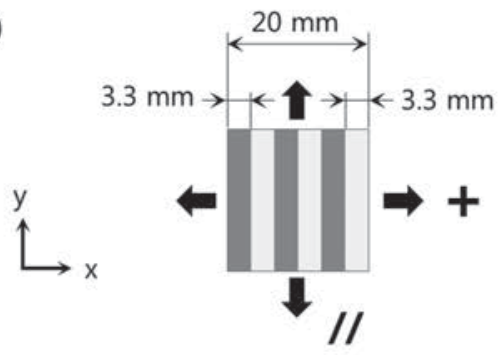

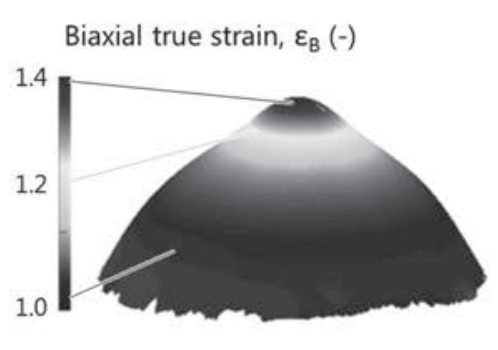
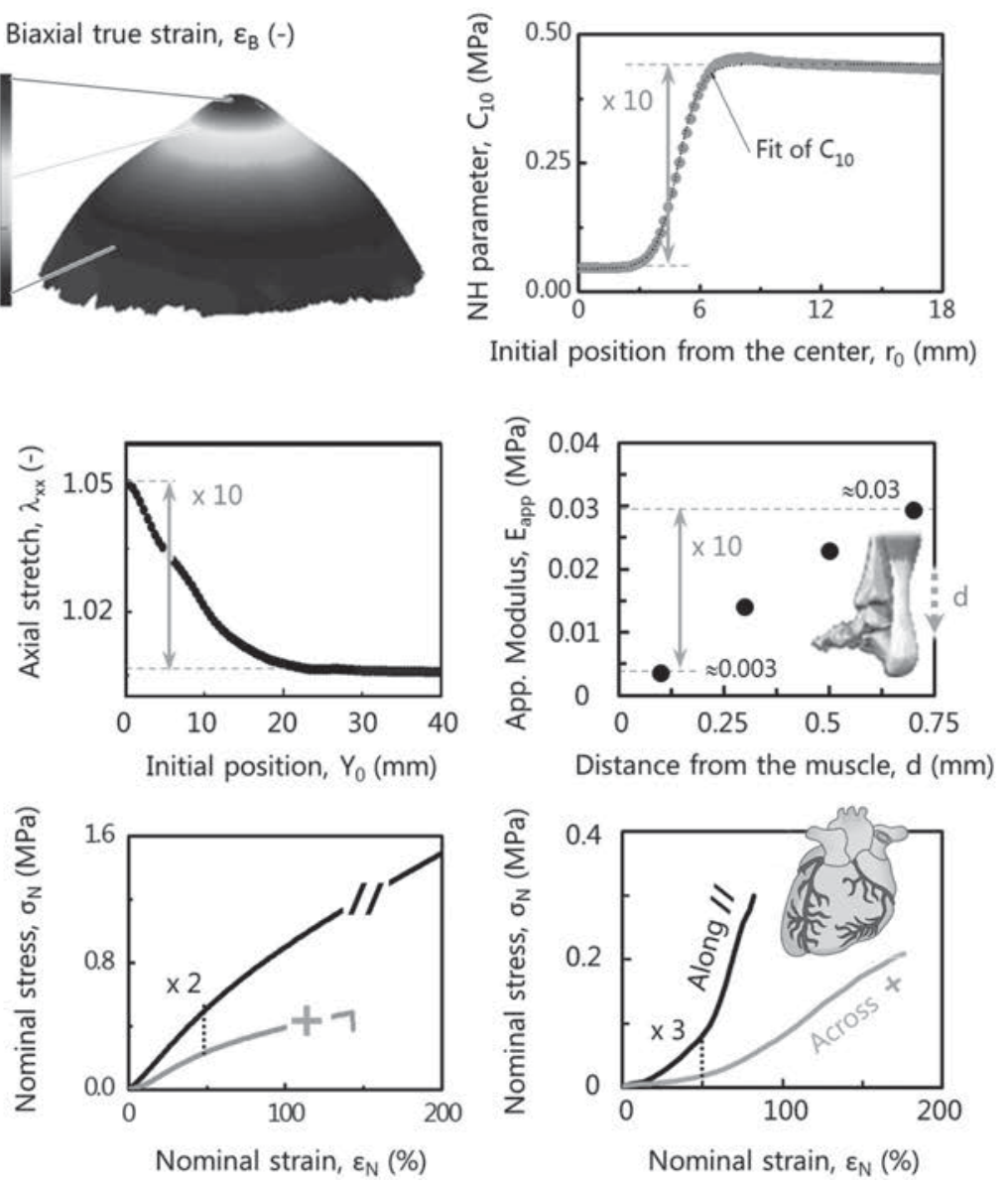

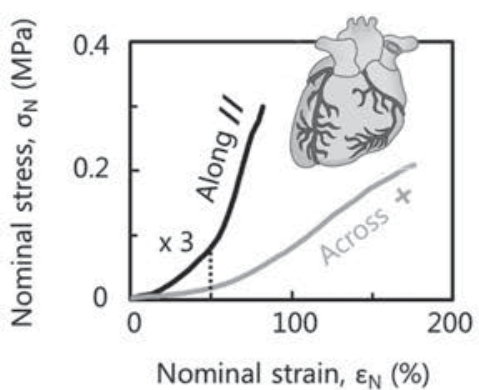

Figure 3. Local control of mechanical properties: a) (Left schematic) Patterned membrane with a 10 ratio macroscopic gradient and (right figure) strain profile at a $5 \%$ macroscopic strain. For comparison, the mechanical properties of human ligament-bone insertion site is provided on the right hand side (adapted from ref. ${ }^{[37]}$ ). b) (Left schematic) Stripped membrane with controlled anisotropy, and (center figure) its behavior in uniaxial tension along the $X$ (Lower-bound configuration) and $Y$-axis (upper-bound configuration). A qualitative comparison with heart tissue is provided in the right figure (modified from ref. ${ }^{[38]}$ ). c) (Left figure) Experimental setup used for interface characterization through bulge test (center figure) strain field determination (right figure) membrane modulus variation with respect to the distance from the axisymmetric axis.

with the setup axis of symmetry. Unlike a simple uniaxial tension test, the present setting prevents lateral contraction. Hence, the strain state is well-defined, axisymmetric, and dependent on the radial coordinate. Finally, the determination of the neo-Hookean parameter $\left(C_{10}\right)$ was performed following the protocol described by Machado et al. ${ }^{[33]}$ The evolution of the $C_{10}$ parameter, which is in good accordance with the one found in bulk materials, allowed for the identification of the interphase's width (Figure 3a). Presently, with our irradiation setup, the interphase is about $3 \mathrm{~mm}$ wide. This interphase width can be easily tuned by adapting the membrane processing condition, for instance, by using focused UV sources, or by diminishing the space between the masks and the irradiated polymer.

Natural soft tissues such as mussel byssus, ${ }^{[34]}$ mammal sinews, ${ }^{[35]}$ or finger extensor apparatus ${ }^{[36]}$ exhibit a macroscopic spatial variation of their mechanical properties. Thanks to this local control of its cross-linking density, the macroscopic properties of the silicone membrane prepared here can be easily tailored. The developed method allows the easy processing of such graded structures, by varying the dose received all along the membrane, as represented in Figure $3 \mathrm{~b}$, where the membrane's strain increases tenfold depending on its position along the $Y$-axis. Such variation, though on different stress and strain levels, is comparable to the one reported for the anterior cruciate ligament-bone insertion site, for instance. ${ }^{[37]}$ Anisotropic membranes can be obtained using a simple striped pattern, as depicted in Figure 3c, and Movies S2 and S3 (Supporting Information). With a stiffness ratio $R$ of 10 between hard and soft zones and a 1 to 1 volumetric ratio, stripe-patterned membrane displays an anisotropic factor of $E_{/ /} / E_{+}=2.3$, close to 3 theoretically expected. Here, $E_{+}$and $E_{/ /}$correspond to the Reuss (lower bound) and Voigt (upper bound) models, 
(a)

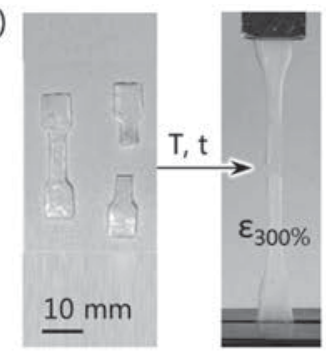

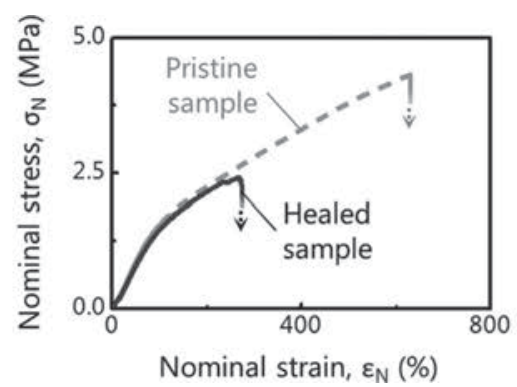

(b)

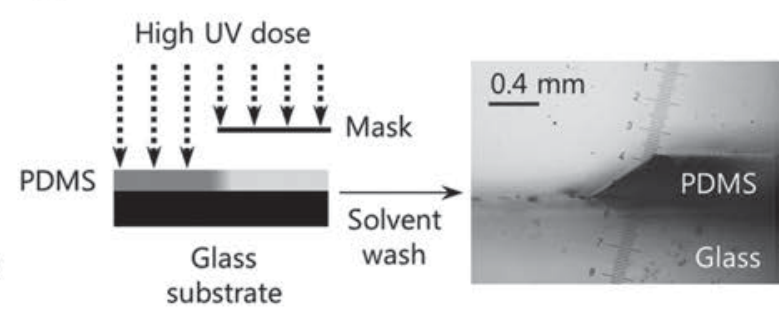

Figure 4. Extensions of the developed process: a) Topological patterning of silicone membranes, via severe UV irradiation and solvent wash of un-cross-linked zones. b) Silicone-silicone gluing of two irradiated zones, via a temperature post curing.

respectively, that are based on the rule of mixture and neglect the contribution of the interfaces. ${ }^{[38]}$ Experimentally, the two moduli were reduced from the macroscopic loads and displacements measured during the test. It is worth noting that the ratio obtained is remarkable for a bulky, monophasic material, and is close to many natural tissues such as heart myocardium ${ }^{[39]}$ or blood vessels. ${ }^{[40,41]}$

The fact that irradiated samples are constituted of a lightly cross-linked network blended with a large amount of free chains allows promising developments. For instance, healable silicone can be obtained by joining two parts of lesser cross-linked silicone membranes, and then heating them, as displayed in Figure $4 \mathrm{a}$ and Movie S4 (Supporting Information). The two networks interpenetrate one another, and the additional thermal curing step finishes cross-linking the silicone, forming a cohesive interface between them. In the case of bio-implantable devices, temperature healable and "patchwork" tailoring of prosthesis can therefore be performed easily, without any additives.

This process also offers the ability to fully block the cross-linking in selected areas, through high irradiation doses and careful curing conditions. The polymer is hence constituted only of free chains, which remains soluble and washable by a solvent within the irradiated zones, while it cross-links normally elsewhere (Figure $4 \mathrm{~b}$ ). This allows for the topographical patterning of thin layers of silicone, which could easily find applications in the field of microfluidic engineering.

\section{Conclusions}

To conclude, we have explored in this study the potential of the photo-instability of a cross-linking catalyst to process biocompatible, transparent silicone membranes with local control of their mechanical and topological properties. The simple, rapid, and versatile approach is adaptable to any commercial silicone elastomer cross-linked by polyaddition, allowing for the design of advanced architectured PDMS membranes based on a unique macromolecular network. The UV-driven mechanism was explored thoroughly so that many applications were identified (gluing, patterning, and grading). The process also gives the possibility to functionalize under-cross-linked zone prior to an additional post-cure of the material, for instance, to craft membranes with different physical properties (surface wetting properties, double cross-linking systems). Focusing on the "structural" performance, difference in modulus as high as 15, along with an infinite choice of architectures can be used to meet the requirements/needs of numerous fields of applications, such as prosthesis engineering or microfluidic.

\section{Supporting Information}

Supporting Information is available from the Wiley Online Library or from the author.

Acknowledgements: This work was supported by the ANR under the project SAMBA (ANR-12-BS09-000). Many thanks to Cécile Chamignon for HR-MAS NMR advice and experiments, to the interns Quentin Barnes and Elodie Henrat for their general help, and to Jean-Pierre Pascault for helpful discussions. Dr. Renaud Rinaldi also thanks Prof. F. W. Zok from UCSB for access to his testing lab, and Kirk Fields for assistance.

Received: May 18, 2016; Revised: July 12, 2016; Published online: ; DOI: 10.1002/mame.201600237

Keywords: elastomer; materials by design; PDMS; UV-light

[1] C. R. McMillin, Rubber Chem. Technol. 2006, 79, 3.

[2] J. Heiner, B. Stenberg, M. Persson, Polym. Test. 2003, 22, 3.

[3] B. Arkles, CHEMTECH 1983, 13, 9.

[4] A. N. Natali, E. L. Carniel, H. Gregersen, Med. Eng. Phys. 2009, 31, 9.

[5] H. Abé, K. Hayashi, S. Masaaki, Data Book on Mechanical Properties of Living Cells, Tissues and Organs, Springer, Tokyo 1996.

[6] R. B. Martin, J. Ishida, J. Biomech. 1989, $22,5$.

[7] M. E. Weber, M. R. Kamal, Polym. Compos. 1992, 13, 2. 
[8] T. Courtney, M. S. Sacks, J. Stankus, J. Guan, W. R. Wagner, Biomaterials 2006, 27, 19.

[9] Y. Wang, Y. Wang, H. Zhang, L. Zhang, Macromol. Rapid Commun. 2006, 27, 14.

[10] J. Crowe-Willoughby, K. L. Weiger, A. E. Özçam, J. Genzer, Polymer 2010, 51, 3.

[11] K. U. Claussen, R. Giesa, T. Scheibel, H. W. Schmidt, Macromol. Rapid Commun. 2012, 33, 3.

[12] R. Libanori, R. M. Erb, A. Reiser, H. LeFerrand, M. J. Süess, R. Spolenak, A. R. Studart, Nat. Commun. 2012, 3, 1265.

[13] L. Meunier, G. Chagnon, D. Favier, L. Orgéas, Eur. J. Mech. A 2015, 50, 87.

[14] M. Skouras, B. Thomaszewski, B. Bickel, M. Gross, Eurographics 2012, 31, 2012.

[15] G. Machado, G. Chagnon, D. Favier, Mech. Mater. 2012, 50, 70.

[16] M. Rebouah, G. Machado, G. Chagnon, D. Favier, Mech. Res. Commun. 2013, 49, 36.

[17] A. A. S. Bhagat, P. Jothimuthu, I. Papautsky, Lab Chip 2007, $7,9$.

[18] D. P. J. Cotton, A. Popel, I. M. Graz, S. P. Lacour, J. Appl. Phys. 2011, 109, 5.

[19] Y. Sun, L. T. Jiang, R. Okada, J. Fu, Langmuir 2012, 28, 29.

[20] B. Marciniec, Comprehensive Handbook on Hydrosilylation, Elsevier, Amsterdam 2013.

[21] B. Karstedt, Patent US3715334 A, 1973.

[22] P. Hitchcock, M. Lappert, N. J. W. Warhurst, Angew. Chem., Int. Ed. 1991, 30, 4.

[23] B. Faust, Mod. Chem. Tech., RSC, London 1997.

[24] L. Meunier, G. Chagnon, D. Favier, L. Orgeas, P. Vacher, Polym. Test. 2008, 27, 6.
[25] A. Romeo, S. P. Lacour, Extreme Mech. Lett. 2015, 3, 1.

[26] A. Stricher, R.G. Rinaldi, C. Barrès, F. Ganachaud, L. Chazeau, RSC Adv. 2015, 5, 66.

[27] E. Delebecq, F. Ganachaud, Appl. Mater. Interfaces 2012, 4, 7.

[28] E. Gharibshahi, E. Saion, Int. J. Mol. Sci. 2012, 13, 11.

[29] Y. Zhou, C. Y. Wang, Y. R. Zhu, Z. Y. Chen, Chem. Mater. 1999, $11,9$.

[30] L. N. Lewis, N. Lewis, J. Am. Chem. Soc. 1986, 108, 23.

[31] J. Stein, L. N. Lewis, Y. Gao, R. A. Scott, J. Am. Chem. Soc. 1999, $121,15$.

[32] J. Li, N. Congbai, J. Peng, Y. Deng, G. Zhang, Y. Bai, C. Ma, W. Xiao, G. Lai, Appl. Organomet. Chem. 2014, 28, 6.

[33] G. Machado, G. Chagnon, D. Favier, Mech. Mater. 2010, $42,9$.

[34] J. H. Waite, H. C. Lichtenegger, G. D. Stucky, P. Hansma, Biochemistry 2004, 43, 24.

[35] R. I. Sharma, J. G. Snedeker, Biomaterials 2010, 31, 30.

[36] K. Qian, K. Traylor, S. W. Lee, B. Ellis, J. Weiss, D. Kamper, J. Biomech. 2014, 47, 12.

[37] J. P. Spalazzi, K. D. Costa, S. B. Doty, H. H. Lu, Trans. Orthop. Res. Soc. 2004, 29, 1271.

[38] R. J. Young, P. A. Lovell, in Introduction to Polymers, 2nd ed., Chapman and Hall, New York 1991.

[39] K.-I. Jang, H. U. Chung, S. Xu, C. H. Lee, H. Luan, J. Jeong, H. Cheng, G.-T. Kim, S. Y. Han, J. W. Woo, Nat. Commun. 2015, 6, 6566.

[40] G. C. Engelmayr, M. Cheng, C. J. Bettinger, J. T. Borenstein, R. Langer, L. E. Freed, Nat. Mater. 2008, 7, 12.

[41] J. D. Humphrey, Cardiovascular Solid Mechanics: Cells, Tissues, and Organs, Springer Science \& Business Media, New York 2002. 